\title{
Order Verification of a Bridgman Furnace Front Tracking Model in Steady State
}

\author{
Robin P. Mooney ${ }^{1}$, Shaun McFadden ${ }^{1}$ \\ ${ }^{1}$ Trinity College Dublin, Dublin 2, Ireland
}

Corresponding author: $\quad$ Tel.: $\quad+353-1-8961359 \quad$ Fax: $\quad+353-1-6795554$

E-mail address: $\quad$ shaun.mcfadden@tcd.ie (S. McFadden)

\begin{abstract}
Numerical model verification is a prerequisite to model validation. However, verification can be difficult if an analytical solution is not available for the normally complex problem that the numerical model is setting out to address. This article aims to formally verify a Bridgman furnace solidification front tracking model code in a steady state scenario. To do this an order verification procedure is applied using an established analytical solution from the literature. The model is verified as first order accurate in space for steady state Bridgman solidification.
\end{abstract}

\section{Highlights}

- A Bridgman furnace front tracking model code is verified for a steady-state solution.

- An order verification procedure is outlined and demonstrated.

- The model is verified as first order accurate in space.

\section{Keywords}

Order verification; Bridgman furnace; front tracking model; solidification; steady state

\section{Introduction}

The word verification (from Latin, verus, meaning true) is defined as the act of demonstrating truth or correctness by comparison to fact, theory, or statement [1]. In numerical modelling, verification refers to the process by which one demonstrates that a partial differential equation (PDE) code correctly solves its governing equations [2]. This process involves comparison of numerically simulated results with a known analytical (exact) solution to the PDE. The numerical model is verified if this comparison is adequately close. In other words, the numerical model accurately solves the equations that constitute the 
mathematical model. Model verification is not to be confused with model validation. Boehm [3] and Blottner [4] define verification as "solving the equations right", and validation as "solving the right equations". Model validation should be carried out after successful model verification, and usually involves comparison of numerically simulated data with experimental data, in order to confirm that the PDE being solved is representative of the real system being modelled. Note that there are differences in opinion regarding these definitions in the literature and, for the purposes of this article, we have adopted the methodology of Roache [5].

A formal procedure for model verification exists [6] where the order of accuracy observed in results from the numerical model is compared to the theoretical order of accuracy of the discretised PDE. Using this procedure, the numerical model is verified when it is shown to be consistent and convergent, and when the observed order of accuracydetermined by comparing simulated results with analytical results - matches the theoretical order of accuracy for the governing PDE.

Bridgman furnaces are used to directionally solidify materials where it is desirable to control growth rate and temperature gradient. The Bridgman solidification procedure involves moving a crucible through a fixed temperature gradient imposed by two heaters that are separated by an adiabatic zone. The process was originally designed [7] and modified [8] to grow single crystal materials in the early $20^{\text {th }}$ century. Now the method it is widely used in research [9] and industry [10].

A front tracking model (FTM) refers to a numerical model that estimates and follows the growth of a solidification interface, or grain envelope, during solidification. FTMs have been used to track the solidification interface in simple phase change problems involving pure materials [11], and also in more complex problems involving binary alloys [12], for example, where columnar dendritic growth occurs [13], or to predict columnar to equiaxed transition [14]. In this paper, we will verify the Bridgman furnace FTM (or BFFTM) of Mooney et al. [15], for steady state Bridgman solidification of high purity titanium.

\section{Verification Method}

\subsection{Background theory}

The formal procedure that we will use for model verification was first implemented by Steinberg and Roache [16] and is summarised by Knupp and Salari [2]. The method focuses on order of accuracy. Under this method, a numerical model is verified when; the observed order of accuracy from simulated results matches the theoretical order of accuracy of the governing partial differential equation, upon which it is based.

\subsubsection{Theoretical order of accuracy}


The discretisation of the governing PDE will yield the theoretical order of accuracy of the model. The PDE is broken down into estimations of each partial derivative using a truncated Taylor series expansion for each derivative term, yielding simple finite difference equations. The truncation error is the difference between the actual value for the derivative term and the estimated value using the difference equations, and can readily be written in terms of the grid resolution. One can then state the theoretical order of accuracy of the discretisation method by looking at the power to which the grid resolution is held in the leading term of the truncation error. For example, considering a one-dimensional domain with grid resolution $\Delta x$, the Taylor series expansion for a second order partial derivative of the dependant variable $T$, in respect of the spatial variable $x$, is given by;

$$
\left.\frac{\partial^{2} T}{\partial x^{2}}\right|_{i}=\underbrace{\frac{T_{i-1}-2 T_{i}+T_{i+1}}{(\Delta x)^{2}}}_{\text {approximaiton }}+\underbrace{O(\Delta x)^{2}}_{\text {truncation }},
$$

where subscript ' $i$ ' refers to a central node or control volume location in the domain. The first term on the right hand side (RHS) of Eq. (1) is the finite difference approximation of the second order partial derivative, while the second term on the RHS is the truncation error. The truncation error is the sum of the terms excluded from the Taylor expansion for the second order partial derivative, i.e., $O(\Delta x)^{2}=\left((\Delta x)^{2} / 4 !\right) d^{4} T / d x^{4}+$ higher order terms, as shown by Özış1k [17]. In this case the lowest power of $\Delta x$ in the leading term of the truncation is two; therefore the theoretical order of accuracy for this discretisation method is second order. Where a PDE contains multiple derivative terms, the lowest power of $\Delta x$ in the leading term of the truncation - across all partial derivative terms in the PDE - gives the overall theoretical order of accuracy for the model.

\subsubsection{Observed order of accuracy}

The observed order of accuracy of the model is determined by comparing the simulated results for an arbitrary test problem at two or more grid resolutions with a known analytical solution. The difference between the simulated results and the analytical solution is known as the numerical error, made up primarily by the truncation error (sometimes called discretisation error), plus the round off error associated with the algorithm software, plus any iterative convergence error. Round off errors are usually negligible relative to the truncation error and iterative convergence error occurs only with implicit finite difference solvers that require a statement of solution tolerance [18].

The numerical solution gives a value for the dependant variable $T_{i}^{\text {num }}$ at distinct locations in the modelled domain. The analytical solution - which is continuous - is evaluated at the same locations to give $T_{i}^{\text {exact }}$. The local numerical error $N E_{i}^{\text {local }}$ can be calculated by their difference, at each discrete position;

$$
N E_{i}^{\text {local }}=T_{i}^{\text {exact }}-T_{i}^{\text {num }} .
$$


Where the numerical scheme uses a fixed grid resolution it is useful to calculate the global numerical error $N E^{\text {global }}$ as follows;

$$
N E^{\text {global }}=\sqrt{\frac{1}{N} \sum_{i}^{N}\left(T_{i}^{\text {exact }}-T_{i}^{\text {num }}\right)^{2}}
$$

where $N$ is the total number of control volume or mesh nodes. The observed order of accuracy for the numerical scheme, $p$, is calculated using the global numerical error at two grid resolutions $\Delta x_{1}$ and $\Delta x_{2}$, as follows [2];

$$
p=\frac{\ln \left(\frac{N E_{\Delta x_{1}}^{\text {global }}}{N E_{\Delta x_{2}}^{\text {global }}}\right)}{\ln \left(\frac{\Delta x_{1}}{\Delta x_{2}}\right)}
$$

Note that the model is said to be consistent when the value for $p$ is greater than zero. In other words, the continuum PDE equation is recovered as $\Delta x \rightarrow 0$. Also, the model is convergent when it can be shown that the magnitude of the global numerical error reduces with $\Delta x$.

\subsection{Order verification procedure}

The verification procedure is illustrated by the flowchart in Fig. 1. Firstly, the theoretical order of accuracy of the model is determined via its governing equations and the finite difference scheme used in the model. Secondly, a test problem is designed. This is where the model is constrained to solve the modelling scenario that we are interested in by setting the boundary conditions; Dirichlet, Neumann, or Robin, and other factors required to define the problem.

While the physical properties of the material are required in this step - in order to run the numerical model later - they are arbitrary in terms of the success of the verification procedure itself. Thirdly, an exact solution to the PDE of interest must be found. Following this, the code is run at two different grid resolutions. The results from these simulations are used in the next step to calculate the observed order of accuracy. If the observed order of accuracy does not match the theoretical order of accuracy we must go back through the code to look for coding errors before re-running the code at two grid resolutions, and so on until the code is verified. 


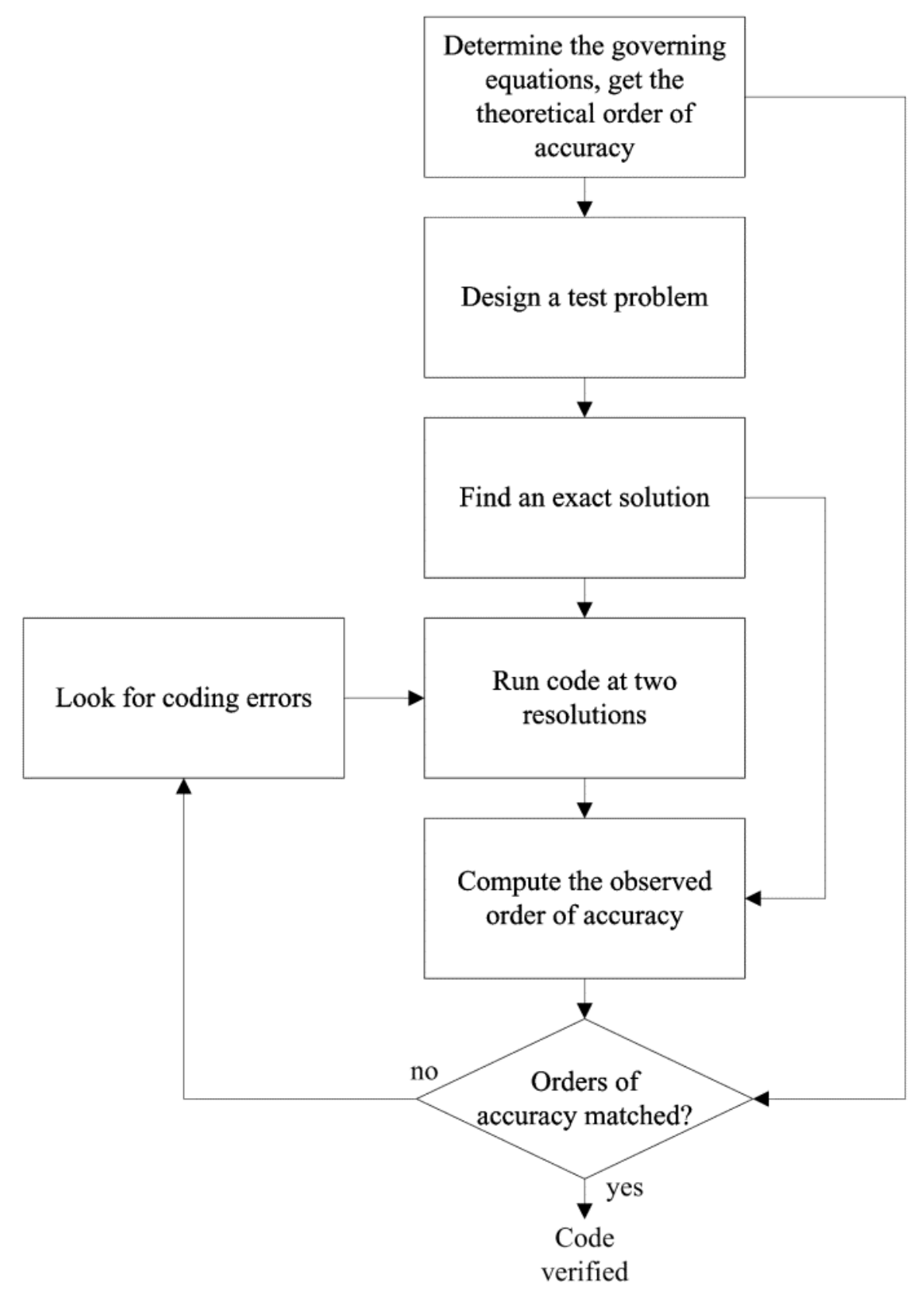

Fig. 1 Code verification procedure, adapted from Knupp and Salari [2].

\section{Bridgman Solidification}

In this section we fully describe the Bridgman furnace solidification procedure. This is followed by details of the particular problem to be solved as part of our verification procedure, i.e., the test problem is defined in terms of boundary conditions, thermophysical properties, and other input data required for modelling.

\subsection{The Bridgman procedure}


A schematic of a Bridgman furnace is shown in Fig. 2. The furnace is tubular and made up of three zones: a hot zone with heater held at a temperature, $T_{H}$, having a heat transfer coefficient with the sample, $h_{H}$; an insulated adiabatic zone (shown hatched) of length, $L_{A}$; and a cold zone with heater held at a temperature, $T_{C}$, having a heat transfer coefficient with the sample, $h_{c}$. Normally, the hot and cold zones have differing heat transfer coefficients (depending on the apparatus), and the hot zone is held at a temperature above the liquidus or melting temperature of the sample material, while the cold zone is held at a temperature below the material solidus or melting temperature. A cylindrical sample with radius, $r$, is contained in a hollow thin walled crucible. Both are translated at a fixed velocity, $u$, through the furnace. The heaters impose a fixed temperature gradient in the sample (long curved line) as it is passed through the furnace.

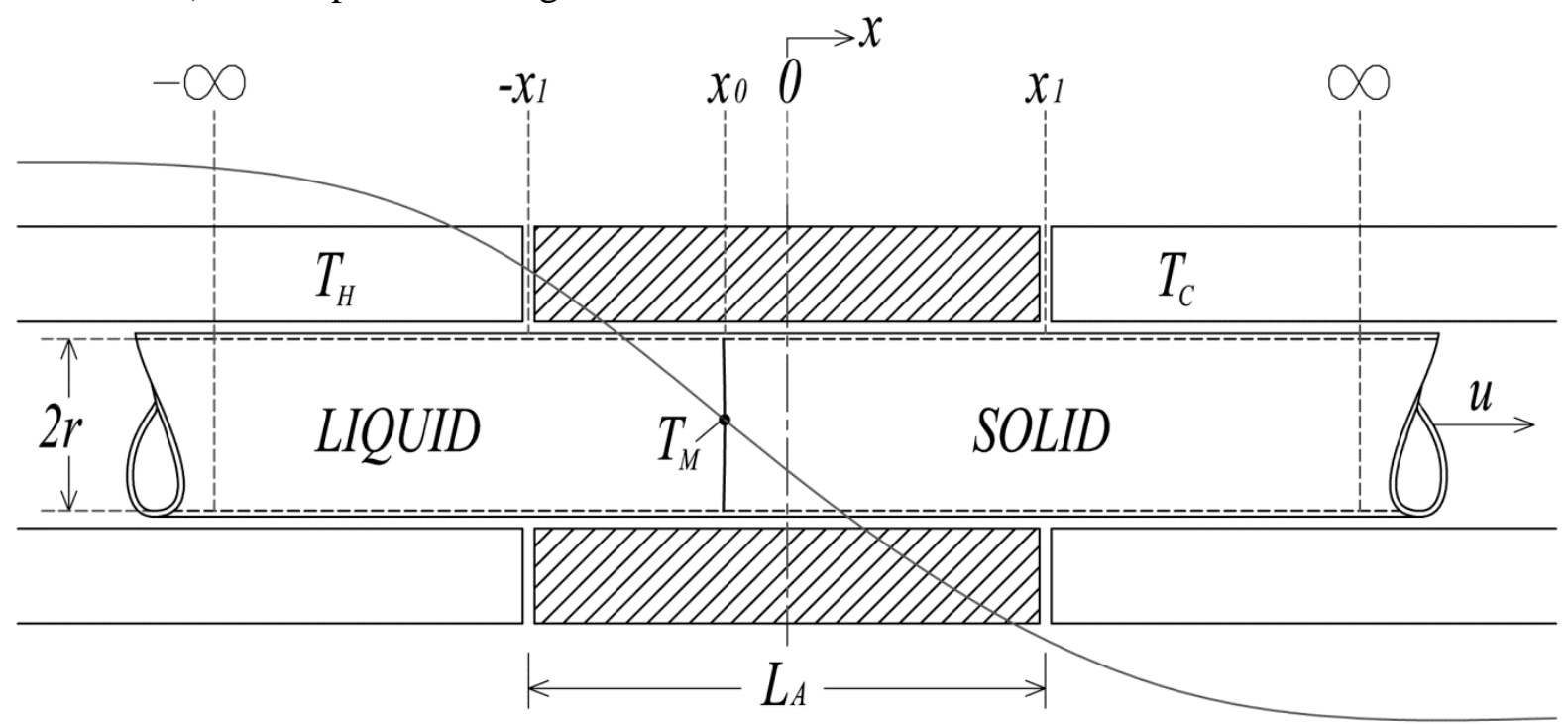

Fig. 2 Schematic of a Bridgman furnace.

A solidification interface is formed at some position in the adiabatic zone where the temperature is equal to the material melting temperature, $T_{M}$. In steady state solidification the position of the interface and the temperature profile is stationary relative to the furnace which is fixed to ground.

Note that the sample and crucible are shown to be infinitely long in the figure. In reality a sample and crucible would have a fixed length, however-for the purposes of developing an analytical model — the rod is treated as infinite.

\subsection{The test problem}

The modelling scenario is that of steady state solidification of a pure material at a constant withdrawal velocity. The material used for modelling purposes was arbitrarily chosen as high purity titanium. Table 1 shows the thermophysical properties for this material and other input data necessary for modelling. 
Table 1 Thermophysical properties for high purity titanium, and other modelling input data

\begin{tabular}{lccl}
\hline Input & Liquid & Solid & Units \\
\hline Thermal conductivity, $k$ & 17 & 17 & $\mathrm{~W} / \mathrm{m}^{\circ} \mathrm{C}$ \\
Density, $\rho$ & 4110 & 4350 & $\mathrm{~kg} / \mathrm{m}^{3}$ \\
Specific heat capacity, $C_{p}$ & 925 & 925 & $\mathrm{~J} / \mathrm{kg}^{\circ} \mathrm{C}$ \\
Heat of fusion, $\Delta H_{f}$ & $2.95 \times 10^{5}$ & $\mathrm{~J} / \mathrm{kg}$ \\
Speed of sound, $v_{\text {sound }}$ & 5090 & $\mathrm{~m} / \mathrm{s}$ \\
Melting temperature, $T_{M}$ & 1670 & ${ }^{\circ} \mathrm{C}$ \\
Hot heat temperature, $T_{H}$ & 1700 & ${ }^{\circ} \mathrm{C}$ \\
Cold heater temperature, $T_{C}$ & 1300 & ${ }^{\circ} \mathrm{C}$ \\
Hot zone heat transfer coefficient, $h_{H}$ & 100 & $\mathrm{~W} / \mathrm{m}^{2}{ }^{\circ} \mathrm{C}$ \\
Cold zone heat transfer coefficient, $h_{C}$ & 600 & $\mathrm{~W} / \mathrm{m}^{2 \circ} \mathrm{C}$ \\
Sample radius, $r$ & 5 & $\mathrm{~mm}$ \\
Adiabatic length, $L_{A}$ & 40 & $\mathrm{~mm}$ \\
Translational velocity, $u$ & 0.4 & $\mathrm{~mm} / \mathrm{s}$ \\
Numerical time step, $\Delta t$ & $0.75 \times 10^{-3}$ & $\mathrm{~s}$ \\
Control volume thickness, $\Delta x$ & $0.8,0.4,0.2,0.1$ & $\mathrm{~mm}$ \\
Modelled domain length, $2 \ell$ & 200 & $\mathrm{~mm}$ \\
\hline
\end{tabular}

\section{Modelling}

In this section a mathematical model of the Bridgman furnace is set out. This is followed by details of an exact analytical solution for that model in the context of the test problem from section 3. Finally, details of the BFFTM to be verified are given, and the theoretical order of accuracy of the model is calculated.

\subsection{Mathematical model}

Considering heat flow in the axial direction only; the heat equation for a long cylindrical rod of uniform cross sectional area $A$, and perimeter $P$, moving at an axial velocity $u$, and transferring heat laterally to the surroundings with a heat transfer coefficient $h$, is given as follows (as adapted from [19] and [20]);

$$
\frac{\partial}{\partial t}\left(\rho C_{p} T\right)=\frac{\partial}{\partial x}\left(k \frac{\partial T}{\partial x}\right)-u \rho C_{p} \frac{\partial T}{\partial x}-\frac{h_{H, C} P}{A}\left(T-T_{H, C}\right)+E
$$

where $\rho, C_{p}$, and $k$ are the density, specific heat capacity, and thermal conductivity of the sample material, respectively; $T_{H, C}$ is the temperature of the surrounding heat source $\left(T_{H}\right)$ or heat sink $\left(T_{C}\right) ; h_{H, C}$ refers to the heat transfer coefficient due to the heat source $\left(h_{H}\right)$, i.e., in the hot region of the furnace, or due to the heat sink $\left(h_{C}\right)$, i.e., in the cold region of the furnace, and finally, $E$ is the latent heat generated per unit volume. This 1-dimensional model with an additional term for peripheral heat loss is deemed appropriate scenarios where the Biot number is less than 0.1. 


\subsection{Analytical solution}

Naumann [21] demonstrated a one dimensional analytical model and solution for Bridgman furnace solidification. With reference to Fig. 2, Naumann's analytical solution is adapted here for our test problem. Assuming steady state heat transfer and using the transformation for dimensionless position, $X=x / r$, where $r$ is the rod radius and $x$ is the real axial position, we can write a dimensionless steady state form of Eq. (5) for equilibrium solidification, as;

$$
\frac{\partial^{2} T}{\partial X^{2}}-P e \frac{\partial T}{\partial X}-2 B i\left(T-T_{H, C}\right)=0
$$

where $P e$ is the Péclet number and $B i$ is the Biot number - as given in Eq. (7) and Eq. (8), respectively — and assuming a characteristic length for the rod equal to its radius [22].

$$
\begin{gathered}
P e=\frac{\rho C_{P} r u}{k} \\
B i=\frac{h_{H, C} r}{k}
\end{gathered}
$$

Assuming a translational velocity $u>0$ the solution to Eq. (6) is given by Eq. (9) through Eq. (12) where $T_{L}(X)$ and $T_{S}(X)$ refer to temperature as a function of dimensionless position in the liquid and solid parts of the rod, respectively.

$$
\begin{array}{ccc}
T_{L}(X)=T_{H}-D \exp \left[\beta^{*}\left(X+X_{1}\right)\right] & \forall & -\infty<X \leq X_{1} \\
T_{L}(X)=A+B \exp \left(P e_{L} X\right) & \forall & -X_{1} \leq X \leq-X_{0} \\
T_{S}(X)=A^{*}+B^{*} \exp \left(P e_{S} X\right) & \forall & X_{0} \leq X \leq X_{1} \\
T_{S}(X)=T_{C}+C \exp \left[-\alpha^{*}\left(X-X_{1}\right)\right] & \forall & X_{1} \leq X<\infty
\end{array}
$$

Where $\alpha, \alpha^{*}, \beta$ and $\beta^{*}$ are constants calculated as follows;

$$
\begin{array}{ll}
\alpha^{*}=\left(\sqrt{4 \alpha^{2}+P e_{S}^{2}}-P e_{S}\right) / 2, & \alpha=\sqrt{2 B i_{C}} \\
\beta^{*}=\left(\sqrt{4 \beta^{2}+P e_{L}^{2}}+P e_{L}\right) / 2, & \beta=\sqrt{2 B i_{H}} .
\end{array}
$$

This solution is valid for a planar solid liquid interface located at a dimensionless position $X_{0}$ within the limits of the adiabatic zone $\pm X_{1}$ only. The thermal resistance of the crucible wall is assumed negligible. Seven unknowns exist; $A, A^{*}, B, B^{*}, C, D$, and $X_{0}$. To solve for these unknowns the following conditions are applied; $T_{L}$ and $d T_{L} / d X$ are continuous at $-X_{1}$, and similarly $T_{S}$ and $d T_{S} / d X$ are continuous at $X_{1}$. It is assumed that the temperature in the liquid is equal to the temperature of the solid at the solid liquid interface, and that solidification occurs in equilibrium at the melting temperature for the material, $T_{M}$. It is 
assumed that the latent heat generated at the liquid-solid interface is equal to the net conduction away from the interface in the solid and liquid phases. In other words, the Stefan condition is applied at the interface. Noting that the temperature gradient in the liquid and solid at the interface are both negative with respect to the coordinate system in Fig. 2, we get the following;

$$
L_{f}=\left.k_{L} \frac{\partial T_{L}}{\partial X}\right|_{X_{0}}-\left.k_{S} \frac{\partial T_{S}}{\partial X}\right|_{X_{0}}
$$

where $L_{f}$ is the rate of latent heat of liberated at the interface per unit area;

$$
L_{f}=\rho_{L} u \Delta H_{f}
$$

and $\Delta H_{f}$ is the latent heat of fusion of the material per unit mass. Finally, the position of the interface, $X_{0}$, is found through an iterative procedure to solve the following transcendental equation;

$$
\frac{k_{L} P e_{L}\left(T_{H}-T_{M}\right) \exp \left(P e_{L} X_{0}\right)}{\exp \left(-P e_{L} X_{1}\right)\left(\frac{P e_{L}}{\beta^{*}}-1\right)+\exp \left(P e_{L} X_{0}\right)}+L_{f}=\frac{k_{S} P e_{S}\left(T_{M}-T_{C}\right) \exp \left(P e_{S} X_{0}\right)}{\exp \left(P e_{S} X_{1}\right)\left(\frac{P e_{S}}{\alpha^{*}}+1\right)-\exp \left(P e_{S} X_{0}\right)}
$$

The remaining terms are calculated as follows;

$$
\begin{gathered}
A=T_{H}+B \exp \left(-P e_{L} X_{1}\right)\left(\frac{P e_{L}}{\beta *}-1\right) \\
A^{*}=T_{C}-B * \exp \left(P e_{S} X_{1}\right)\left(\frac{P e_{S}}{\alpha^{*}}+1\right) \\
B=-\frac{T_{H}-T_{M}}{\exp \left(-P e_{L} X_{1}\right)\left(\frac{P e_{L}}{\beta^{*}}-1\right)+\exp \left(P e_{L} X_{0}\right)} \\
B^{*}=-\frac{T_{M}-T_{C}}{\exp \left(P e_{S} X_{1}\right)\left(\frac{P e_{S}}{\alpha *}+1\right)-\exp \left(P e_{S} X_{0}\right)} \\
C=-P e_{S} B * \frac{\exp \left(P e_{S} X_{1}\right)}{\alpha^{*}} \\
D=-P e_{L} B * \frac{\exp \left(-P e_{L} X_{1}\right)}{\beta^{*}}
\end{gathered}
$$


The problem data in Table 1 was applied to this set of equations. A solution for the front position was computed using the f zero [23] command in Matlab ${ }^{\circledR}$ for solving nonlinear functions. The temperature profile in each section of the furnace could then be determined.

\subsection{Numerical solution}

In this section we outline how the Bridgman furnace front tracking model (BFFTM) of Mooney et al. [15] was implemented to solve our test problem. Then we determine the theoretical order of accuracy of the model. This is followed by details of the growth law used, and the treatment of solid fraction in the solution.

\subsubsection{Implementation of the BFFTM}

The BFFTM was previously demonstrated for a transient solidification problem, involving a binary alloy, where step changes in the withdrawal velocity occurred [15]. It was shown how the model could be used to determine a steady state temperature profile, for a fixed withdrawal velocity, and assuming some arbitrary initial temperature profile in the sample. We applied the model in the same way to determine a steady state solution to our test problem. Firstly, the steady state temperature profile for the test problem was found where the sample was stationary. A step change in withdrawal velocity was then introduced and the evolution of the temperature profile was observed until a steady state was reached. The final temperature profile was then used to compare with the results from the analytical model.

Note that, in the numerical solution, thermal resistance at the crucible wall was assumed to be negligible (as in the analytical solution). Also, Dirichlet boundary conditions were applied to the domain boundaries such that; $T=T_{H}$ at $x=-\ell / 2$, and $T=T_{C}$ at $x=+\ell / 2$. The value for $\ell$ was chosen suitably long enough so that the temperature profile approached $T_{H}$ at the west domain boundary and $T_{C}$ at the east domain boundary, as predicted by the analytical model.

\subsubsection{Theoretical order of accuracy of the BFFTM}

The control volume (CV) approach is used in the BFFTM. The sample is divided into disc shaped CVs, $\Delta x$ metres wide. The governing heat equation, Eq. (5), is integrated over one CV to give the following [17];

$$
\begin{aligned}
\rho C_{p} \frac{\partial T}{\partial t} V_{C V} & =\left\{\left[k \frac{\partial T}{\partial x}\right]_{e}-\left[k \frac{\partial T}{\partial x}\right]_{w}\right\} A-\rho C_{p} u\left[T_{e}-T_{w}\right] A \\
& -\frac{h_{H, C} P}{A}\left(T-T_{H, C}\right) V_{C V}+\rho L \frac{\partial V_{s}}{\partial t}
\end{aligned}
$$


where $V_{C V}$ is the volume of the $\mathrm{CV}$, the subscripts ' $e$ ' and ' $w$ ' refer to the east and west flat faces of the $\mathrm{CV}$ respectively, $L$ is the latent heat generated per unit mass, $V_{s}$ is the volume of solid material in a $C V$, and $\rho L \partial V_{s} / \partial t=E V_{C V}$. Dividing across by $A$, and introducing Taylor series' expansions for the partial derivative terms (except for $\partial V_{s} / \partial t$ ), we get;

$$
\begin{gathered}
\rho C_{p}\left[\frac{\left(T_{i}^{m+1}-T_{i}^{m}\right)}{\Delta t}+O(\Delta t)\right] \Delta x=k\left[\frac{\left(T_{i+1}-T_{i}\right)}{\Delta x}+O(\Delta x)\right] \\
-k\left[\frac{\left(T_{i}-T_{i-1}\right)}{\Delta x}+O(\Delta x)\right]-\rho C_{p} u\left[T_{e}-T_{w}\right]-\frac{h_{H, C} P}{A}\left[T_{i}-T_{H, C}\right] \Delta x+\frac{\rho L}{V_{C V}} \frac{\partial V_{s}}{\partial t} \Delta x,
\end{gathered}
$$

where the superscript ' $m$ ' is the temporal label, and the subscript ' $i$ ' is the spatial label for $\mathrm{CV}$ s lined up in the $x$-direction. Looking at the final term in this equation - that deals with the latent heat released during solidification-note that $V_{s}=g_{s} d$, where $g_{s}$ is the fraction of solid within a $\mathrm{CV}$ and $d$ is the captured volume in a CV, so then $\partial V_{s} / \partial t=g_{s}(\partial d / \partial t)+d\left(\partial g_{s} / \partial t\right)$ giving the following equation when Taylor series' replace the partial derivative terms;

$$
\frac{\partial V_{s}}{\partial t}=g_{s}\left[\frac{\left(d_{i}^{m+1}-d_{i}^{m}\right)}{\Delta t}+O(\Delta t)\right]+d\left[\frac{\left(g_{s i}^{m+1}-g_{s i}^{m}\right)}{\Delta t}+O(\Delta t)\right]
$$

For more detailed accounts of the front tracking algorithm used in the BFFTM see references [13] and [14].

Looking at the truncation terms only, in Eq. (25) we see that the lowest power of $\Delta x$ is one, and in Eq. (26) the lowest power of $\Delta t$ is one. This gives the theoretical order of accuracy of the BFFTM as first order in space and first order in time. By removing the truncation terms, we are using first order approximations of the Taylor's series expansions of the partial derivative terms, i.e., we are using Euler's method. This yields the explicit finite difference scheme used in the BFFTM. Refer to [15] and [24] for a further description of the BFFTM algorithm.

\subsubsection{Growth law for high purity titanium}

The BFFTM, and its predecessors [13] and [14], have been used to simulate dendritic columnar growth of alloys. However, the BFFTM can be adapted for growth of pure materials - where a planar non-dendritic interface occurs - by suitable adjustment of the growth law kinetics. The velocity of the solid liquid interface, during the solidification of a pure material, is directly proportional to the level of kinetic undercooling at the interface [25] as follows;

$$
v=\mu_{k} \Delta T
$$


where $v$ is the interface velocity, $\mu_{k}$ is the attachment kinetics coefficient, and $\Delta T$ is the kinetic undercooling at the interface which is equal to the difference between the material melting temperature and the temperature at the interface. Typically, for pure materials, the level of undercooling is very small $\left(<3{ }^{\circ} \mathrm{C}\right)$ unless solidification occurs at a very high speeds $(100-1000 \mathrm{~m} / \mathrm{s})$. The attachment kinetics coefficient, $\mu_{k}$, was estimated using the collision limited growth model of Turnbull and Bagley [26];

$$
\mu_{k}=\frac{v_{\text {sound }} \Delta H_{f}^{\text {mol }}}{\mathfrak{R} T_{M}^{2}}
$$

where $v_{\text {sound }}$ is the velocity of sound, $\Delta H_{f}^{m o l}$ is the molar latent heat of fusion for the material, $\mathfrak{R}$ is the molar gas constant, and $T_{M}$ is the material melting temperature in units of Kelvin. The modelling data in Table 1 was used to calculate $\mu_{k}$ to be $2.294 \mathrm{~m} / \mathrm{s}^{\circ} \mathrm{C}$.

\subsubsection{Fraction of solid}

The fraction of solid, $g_{s}$, as a function of temperature for pure titanium, was estimated by an approximation to a step change of $0 \rightarrow 1$ near the melting temperature as follows;

$$
g_{s}=\left\{\begin{array}{ccc}
0 & \forall & T \geq T_{M} \\
\frac{T_{M}-T}{\in} & \forall & T_{M}-\in<T<T_{M} \\
1 & \forall & T \leq T_{M}-\in
\end{array},\right.
$$

where $\in$ is the thermal range over which phase change occurs, set to a very low value $(0.01$ ${ }^{\circ} \mathrm{C}$ in our case). This arrangement is equivalent to having a linear solid fraction to temperature relationship for an alloy with a solidification interval of $0.01{ }^{\circ} \mathrm{C}$. This approach will give a result similar to the enthalpy linearization method [27] where enthalpy is varied over a very small temperature range about the melting temperature.

\section{Results}

As per the description in section 4.3.1, the steady state temperature profile for a fixed withdrawal velocity was determined dynamically using the BFFTM. Firstly, the temperature profile for a stationary sample was estimated using the BFFTM, shown as $T_{\text {initial }}$ in Fig. 3. 


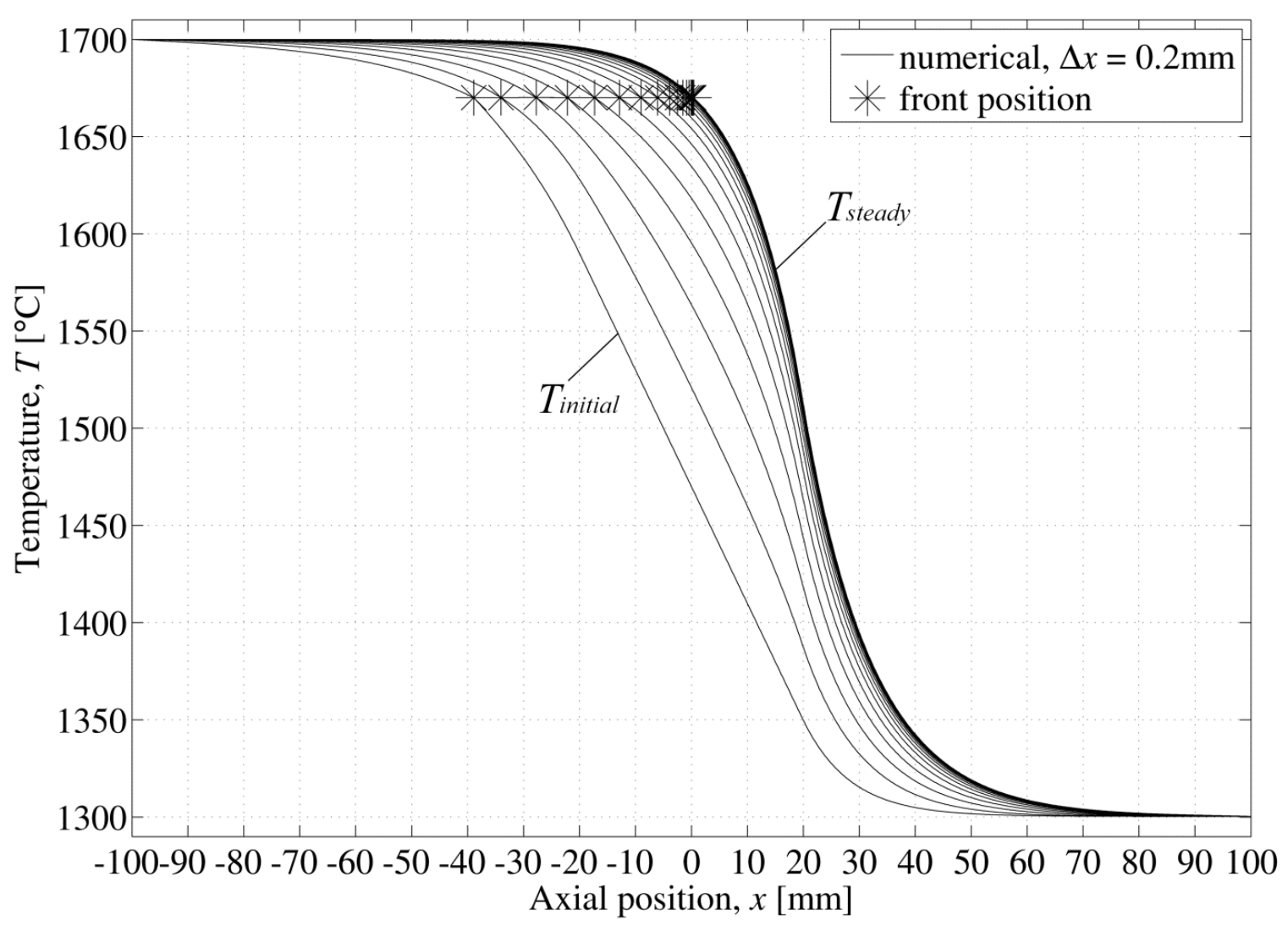

Fig. 3 Evolution of temperature profile resulting from step change in withdrawal velocity

Then a step change in withdrawal velocity was introduced and the evolution of the temperature profile was observed until a steady state was reached, shown as $T_{\text {steady }}$ in Fig. 3 . This figure shows the temperature profile evolution over a $500 \mathrm{~s}$ period with the step change in withdrawal velocity $(u=0 \rightarrow 0.4 \mathrm{~mm} / \mathrm{s})$ introduced at $t>0$. The time between subsequent temperature profiles is $10 \mathrm{~s}$. The front marker for each curve is shown as an asterisk.

Simulations were carried out at four different grid resolutions, as per Table 1, starting at $\Delta x=0.8 \mathrm{~mm}$ and reducing the resolution by a factor of two until $\Delta x=0.1 \mathrm{~mm}$. In each case the resulting steady state temperature profile from the numerical model was compared to the analytical solution and the global numerical error was calculated. The observed order of accuracy was then calculated by comparing results over two consecutive grid refinements. Table 2 shows a summary the results obtained.

Table 2 Verification simulation results

\begin{tabular}{cccc}
\hline Simulation & $\begin{array}{c}\text { Grid resolution, } \\
\Delta x[\mathrm{~mm}]\end{array}$ & $\begin{array}{c}\text { Global numerical error, } \\
N E^{\text {global }}\left[{ }^{\circ} \mathrm{C}\right]\end{array}$ & $\begin{array}{c}\text { Observed order of } \\
\text { Accuracy, } p\end{array}$ \\
\hline$\# 1$ & 0.8 & 2.11 & 0.61 \\
$\# 2$ & 0.4 & 1.38 & 1.07 \\
$\# 3$ & 0.2 & 0.66 & 0.98 \\
\hline 4 & 0.1 & 0.33 & \\
\hline
\end{tabular}




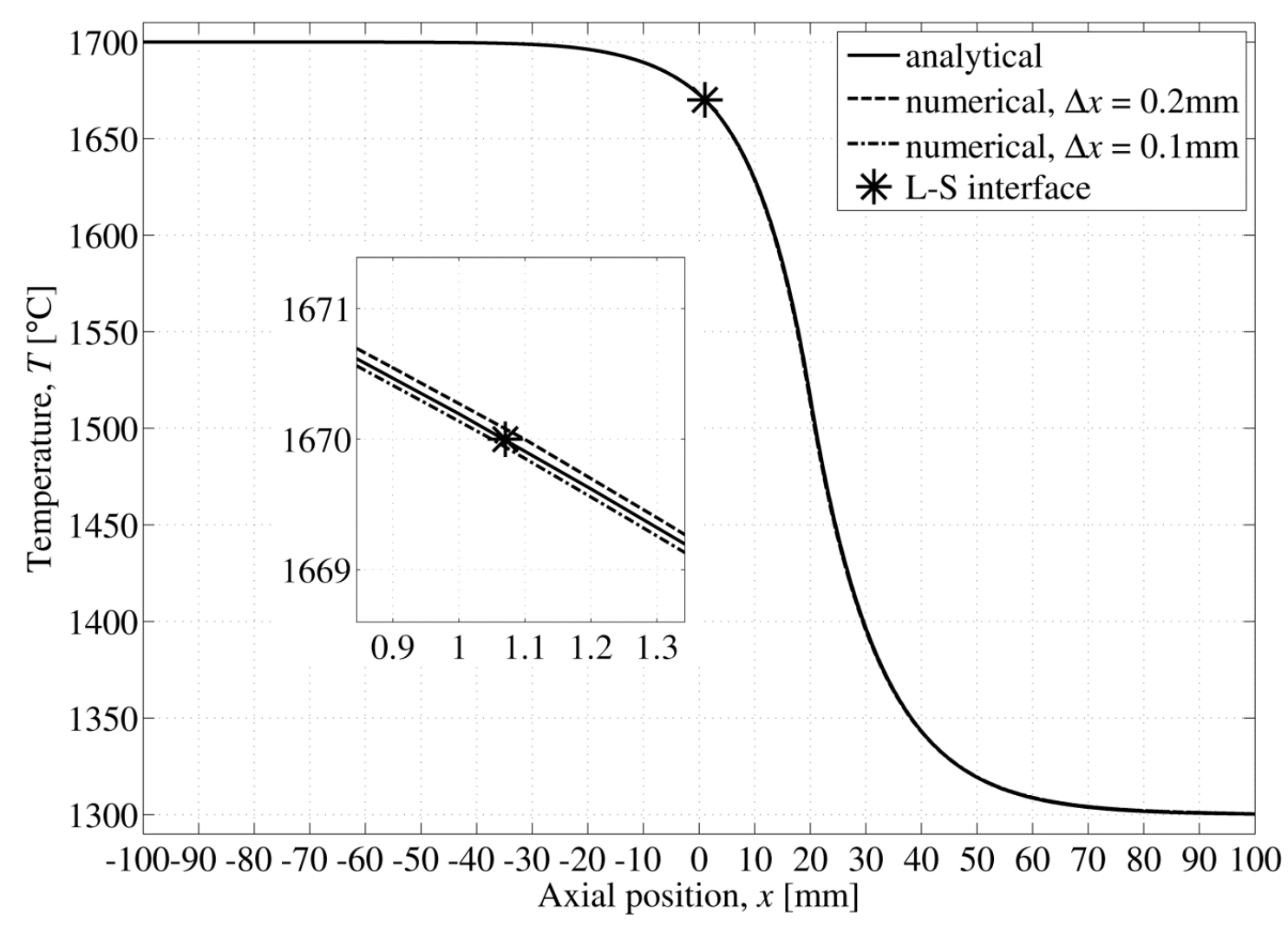

Fig. 4 Temperature profile for comparison for analytical and numerical solutions

The temperature profiles obtained in simulation \#3 $(\Delta x=0.2 \mathrm{~mm})$ and simulation \#4 $(\Delta x=0.1 \mathrm{~mm})$ are shown with the analytical solution in Fig. 4 . The liquid-solid interface location, as predicted this time by the analytical model, is shown by an asterisk.

\section{Discussion}

The discussion is limited to spatial order of accuracy verification since there was no transient analytical solution available to verify the temporal order of accuracy of the model. In Table 2 we see that the observed order of accuracy, obtained by comparing simulations at successively refined grid resolutions, converged to within two significant digits of the spatial theoretical order of accuracy for the model, i.e., it converged to one (first order). The model used a numerically consistent finite difference scheme, since $p>0$. The results show that the model was asymptotically convergent given that the global numerical error was tending to zero as $\Delta x$ was reduced. Also, we can say that the first term in the truncation error dominated the higher order terms, given that value for $p$ was shown to be converging [2]. The global numerical error converged by a factor of approximately two during successive grid refinements. This was to be expected given that the refinement ratio is 2 and that $p$ was approximately equal to 1 . If, for example, $p$ was approximately equal to 2 , i.e., second order 
accuracy, we would have expected the numerical error to reduce by approximately a factor of 4 since, $N E \propto(\Delta x)^{p}$.

The source of the error was most certainly due to the truncation of the derivative estimates in the discretisation scheme only. Round-off error was negligible, given that the code was implemented in double precision, thereby assigning 16 significant digits for storage of all numerically processed variables. Also the numerical scheme required no iterative procedure so there was no potential for iterative convergence error in the numerical scheme. The analytical model was solved, however, using an iterative procedure-specifically Eq. (17) in Matlab ${ }^{\circledR}$ _ but the error tolerance was negligible at $2.22 \times 10^{-16}$.

The truncation error could have been reduced by adding more terms to the Taylor series approximations of the partial derivative terms in the discretisation scheme. Alternatively, the existing first-order Taylor series approximation could have been improved using numerical techniques such as Heun's method or the midpoint method [18]. Either of these improvements would increase the order of accuracy of the model to at least second order.

In Fig. 4 we can see the analytical temperature profile compared with the numerically estimated temperature profiles for simulation \#3 $(\Delta x=0.2 \mathrm{~mm})$ and simulation $\# 4\left(\Delta x_{2}=0.1\right.$ $\mathrm{mm})$. It is clear that the numerical solution was tending towards the analytical solution as the grid resolution was reduced. The maximum local error observed in simulation \#3 was approximately $0.2 \%$ of the temperature range, and $0.1 \%$ of the temperature range in simulation \#4. This gave an accuracy of $0.8^{\circ} \mathrm{C}$ at a grid resolution of $0.2 \mathrm{~mm}$, and $0.4{ }^{\circ} \mathrm{C}$ at a grid resolution of $0.1 \mathrm{~mm}$, over a $400^{\circ} \mathrm{C}$ temperature range. The simulated front position was within $0.04 \mathrm{~mm}$ of the analytical prediction for simulation \#3, i.e., $0.02 \%$ of the domain size, and $0.02 \mathrm{~mm}$ for simulation \#4, i.e., $0.01 \%$ of the domain size.

It should be noted that the analytical model assumes equilibrium solidification occurs. In other words, the liquid-solid interface temperature was fixed at the equilibrium melting temperature during solidification. While the BFFTM assumed that non-equilibrium solidification occurs. In the latter case the front at some temperature below the equilibrium melting temperature during solidification, i.e., it was assumed to be undercooled. Since the material solidifying is pure, this is a kinetic undercooling which is normally very small at low solidification velocities. For example, in our case with $u=0.4 \mathrm{~mm} / \mathrm{s}$ the undercooling is approximately equal to $2 \times 10^{-4}{ }^{\circ} \mathrm{C}$. This would account for minor differences between the predicted front location in the analytical and numerical model results. For example, given the temperature gradient at the solidification front (from the steady state temperature profile) of $2739^{\circ} \mathrm{C} / \mathrm{m}$, this equated to a difference of $7.3 \times 10^{-4} \mathrm{~mm}$ between numerical and analytical front positions.

Finally, in the BFFTM model, it was necessary to model the phase change occurring over a small but finite temperature range. This feature of the model is a numerical artefact whose effect on the results should be minimal. An exercise was carried out to investigate what effect increasing the range of temperature over which phase change occurs, $\in$, had on order accuracy. The observed order of accuracy, $p$, was calculated by comparing numerical results at a grid resolution of $0.2 \mathrm{~mm}$ and $0.1 \mathrm{~mm}$ and by increasing the thermal range, $\in$, from 0.01 to $0.5^{\circ} \mathrm{C}$. Table 3 gives the results from this exercise where it can be seen that first order accuracy was lost above a thermal range of $0.1{ }^{\circ} \mathrm{C}$. The order verification procedure 
was non convergent when the thermal range was set to $0.5^{\circ} \mathrm{C}$. This exercise outlined the sensitivity of the method used to calculate $p$ and also showed that a very small thermal range, i.e., less than $0.1^{\circ} \mathrm{C}$, is appropriate for the test problem.

Table 3 Effect of increasing the thermal range for phase change

\begin{tabular}{cc}
\hline Thermal range, $\in\left[{ }^{\circ} \mathrm{C}\right]$ & Observed order of accuracy, $p$ \\
\hline 0.01 & 0.98 \\
0.1 & 1.02 \\
0.25 & 1.13 \\
0.5 & Non-convergent (negative $p$ ) \\
\hline
\end{tabular}

\section{Conclusion}

The BFFTM code of Mooney et al. [15] has been verified for a steady state scenario using the order verification method given by Knupp and Salari [2] by way of demonstrative example, specifically, steady state solidification of high purity titanium. The model is first order accurate in space. The source of numerical error is primarily truncation error. The order of accuracy of the model could be increased by using a higher order interpolation in the discretisation scheme of the model. For the test problem investigated, asymptotic convergence of the global numerical error was observed for grid resolutions of $0.8 \mathrm{~mm}$ or less, and the grid resolution necessary for a maximum local numerical error of $<1{ }^{\circ} \mathrm{C}$ was 0.2 $\mathrm{mm}$. A value of $0.01{ }^{\circ} \mathrm{C}$ was deemed appropriate for the thermal range over which phase change occurred in the numerical model.

\section{Acknowledgement}

This work was carried out as part of the GRADECET (GRAvity DEpendance of Columnar to Equiaxed Transition in Ti-Al Alloys) research project. The authors would like to thank the European Space Agency PRODEX programme for funding the research (agreement number: 4000107132) under the management of the Irish Space Delegation at Enterprise Ireland. Finally, the authors would like to thank Dr. David Browne of University College Dublin for providing useful discussions during the preparation of this article.

$\begin{array}{ll}\text { Nomenclature } \\ A & \text { sample cross sectional area }\left[\mathrm{m}^{2}\right] \\ B i & \text { Biot number } \\ C_{p} & \text { specific heat capacity at constant pressure }\left[\mathrm{J} / \mathrm{kg}^{\circ} \mathrm{C}\right] \\ d & \text { captured volume }\left[\mathrm{m}^{3}\right] \\ E & \text { latent heat per unit volume }\left[\mathrm{J} / \mathrm{m}^{3}\right] \\ g_{s} & \text { solid fraction } \\ h & \text { heat transfer coefficient }\left[\mathrm{W} / \mathrm{m}^{2 \circ} \mathrm{C}\right] \\ k & \text { thermal conductivity }\left[\mathrm{W} / \mathrm{m}^{\circ} \mathrm{C}\right]\end{array}$


half domain length [m]

$L \quad$ latent heat of fusion per unit mass $[\mathrm{J} / \mathrm{kg}]$ (in the numerical solution)

$L_{f} \quad$ rate of latent heat liberated at a moving interface per unit area $\left[\mathrm{W} / \mathrm{m}^{2}\right]$

$L_{A} \quad$ adiabatic zone length [m]

$N \quad$ total number of control volumes

$N E \quad$ numerical error $\left[{ }^{\circ} \mathrm{C}\right]$

$p \quad$ observed order of accuracy

$P \quad$ perimeter $[\mathrm{m}]$

$P e \quad$ Péclet number

$q \quad$ heat flux $\left[\mathrm{W} / \mathrm{m}^{2}\right]$

$\mathfrak{R} \quad$ molar gas constant, $8.314[\mathrm{~J} / \mathrm{molK}]$

$r \quad$ radius $[\mathrm{m}]$

T temperature $\left[{ }^{\circ} \mathrm{C}\right]$

$u \quad$ withdrawal velocity $[\mathrm{m} / \mathrm{s}]$

$v \quad$ interface velocity $[\mathrm{m} / \mathrm{s}]$

$v_{\text {sound }} \quad$ velocity of sound $[\mathrm{m} / \mathrm{s}]$

$V_{s} \quad$ volume of solid in a control volume $\left[\mathrm{m}^{3}\right]$

$V_{C V} \quad$ volume of one control volume $\left[\mathrm{m}^{3}\right]$

$x \quad$ axial position $[\mathrm{m}]$

$X \quad$ dimensionless axial position

$\Delta H_{f} \quad$ latent heat of fusion per unit mass $[\mathrm{J} / \mathrm{kg}]$ (in the analytical solution)

$\Delta t \quad$ time step [s]

$\Delta T \quad$ undercooling $\left[{ }^{\circ} \mathrm{C}\right]$

$\Delta x \quad$ CV thickness [m]

$\mu_{k} \quad$ attachment kinetics coefficient $\left[\mathrm{m} / \mathrm{s} \cdot{ }^{\circ} \mathrm{C}\right]$

$\rho \quad$ density $\left[\mathrm{kg} / \mathrm{m}^{3}\right]$

\section{Sub/Superscripts}

$0 \quad$ liquid-solid interface

$e \quad$ east face

C cold region

$H \quad$ hot region

$i \quad$ spatial label

$L \quad$ liquid

M melting

$m \quad$ temporal label

num numerical

$S \quad$ solid

w west face

\section{References}


[1] Oxford University Press, "verification, n.", OED Online. http://www.oed.com/view/Entry/222504. [Accessed: 08-Aug-2013].

[2] P. Knupp, K. Salari, Verification of Computer Codes in Computational Science and Engineering, CRC Press, Boca Raton, 2003.

[3] B.W. Boehm, Software Engineering Economics, Prentice-Hall, New Jersey, 1981.

[4] F.G. Blottner, Accurate Navier-Stokes results for the hypersonic flow over a spherical nosetip, J. Spacecr. Rockets. 27 (1990) 113-122. doi:10.2514/3.26115.

[5] P.J. Roache, Verification of Codes and Calculations, AIAA J. 36 (1998) 696-702. doi:10.2514/2.457.

[6] P.J. Roache, Verification and Validation in Computational Science and Engineering, 1st ed., Hermosa, 1998.

[7] P.W. Bridgman, Certain physical properties of single crystals of tungsten, antimony, bismuth, tellurium, cadmium, zinc and tin, Proc. Am. Acad. Arts Sci. 60 (1925) 303. doi: $10.2307 / 25130058$.

[8] D.C. Stockbarger, The production of large single crystals of lithium fluoride, Rev. Sci. Instrum. 7 (1936) 133-136. doi:10.1063/1.1752094.

[9] K.A. Jackson, J.D. Hunt, Transparent compounds that freeze like metals, Acta Metall. 13 (1965) 1212-1215. doi:10.1016/0001-6160(65)90061-1.

[10] A. Kermanpur, N. Varahraam, E. Engilehei, P. Mohammadzadeh, M. Davami, Directional solidification of Ni base superalloy IN738LC to improve creep properties, Mater. Sci. Technol. 16 (2000) 579-586. doi:10.1179/026708300101508117.

[11] C. Li, S. Garimella, J. Simpson, Fixed-grid front-tracking algorithm for solidification problems, part I: Method and validation, Numer. Heat Transf. Part B-Fundamentals. 43 (2003) 117-141. doi:10.1080/713836172.

[12] J. Banaszek, P. Furmański, M. Rebow, Modelling of transport phenomena in cooled and solidifying single component and binary media, Oficyna Wydawnicza Politechniki Warszawskiej, 2005.

[13] D.J. Browne, J.D. Hunt, A Fixed Grid Front-Tracking Model of the Growth of a Columnar Front and an Equiaxed Grain During Solidification of an Alloy, Numer. Heat Transf. Part B-Fundamentals. 45 (2004) 395-419. doi:10.1080/10407790490430606.

[14] S. McFadden, D.J. Browne, A front-tracking model to predict solidification macrostructures and columnar to equiaxed transitions in alloy castings, Appl. Math. Model. 33 (2009) 1397-1416. doi:10.1016/j.apm.2008.01.027.

[15] R.P. Mooney, S. McFadden, M. Rebow, D.J. Browne, A Front Tracking Model for Transient Solidification of $\mathrm{Al}-7 \mathrm{wt} \% \mathrm{Si}$ in a Bridgman Furnace, Trans. Indian Inst. Met. 65 (2012) 527-530. doi:10.1007/s12666-012-0201-2.

[16] S. Steinberg, P.J. Roache, Symbolic manipulation and computational fluid dynamics, J. Comput. Phys. 57 (1985) 251-284. doi:10.1016/0021-9991(85)90045-2.

[17] M.N. Özışık, Finite difference methods in heat transfer, 1st ed., CRC Press, Boca Raton, 1994.

[18] S.C. Chapra, R.P. Canale, Numerical Methods for Engineers, 6th ed., McGraw Hill, 2010.

[19] H.S. Carslaw, J.C. Jaeger, Conduction of Heat in Solids, 2nd ed., Oxford University Press, London, 1959.

[20] A. Bejan, Heat Transfer, 1st ed., John Wiley \& Sons, New York, 1993.

[21] R.J. Naumann, An analytical approach to thermal modeling of bridgman-type crystal growth: II. Two-dimensional analysis, J. Cryst. Growth. 58 (1982) 569-584. doi:10.1016/0022-0248(82)90144-0. 
[22] F.P. Incropera, D.P. Dewitt, T.L. Bergman, A.S. Lavine, Fundamentals of Heat and Mass Transfer, 6th ed., John Wiley \& Sons, New York, 2007.

[23] MathWorks, "fzero, Root of nonlinear function", http://www.mathworks.co.uk/help/matlab/ref/fzero.html. [Accessed: 12-Aug-2013].

[24] R.P. Mooney, S. McFadden, Z. Gabalcová, J. Lapin, An experimental-numerical method for estimating heat transfer in a Bridgman furnace, Appl. Therm. Eng. 67 (2014) 61-71. doi:10.1016/j.applthermaleng.2014.02.048.

[25] J.A. Dantzig, M. Rappaz, Solidification, 1st ed., EPFL Press, Lausanne, 2009.

[26] D. Turnbull, B.G. Bagley, Treatise on Solid State Chemistry, Vol. 5: Changes of state, Plenum, New York, 1975.

[27] V.R. Voller, An Overview of Numerical Methods for Solving Phase Change Problems, in: W.J. Minkowycz, E.M. Sparrow (Eds.), Adv. Numer. Heat Transf. Vol. 1, CRC Press, 1997: pp. 341-380. 